\title{
A New Type of Magnetic Activity Forecast for High Geomagnetic Latitudes
}

\author{
Jaroslava HRUSKA and Richard L. COLES \\ Geophysics Division, Geological Survey of Canada, 1 Observatory Crescent, \\ Ottawa, Canada, K1A $0 Y 3$
}

(Received February 2, 1987)

\begin{abstract}
A new multi-zone concept for forecasting magnetic activity at high geomagnetic latitudes (above $55^{\circ} \mathrm{N}$ ), resulting in more definitive medium-term forecasts (for up to 27 days in advance) for the Canadian region, shows good agreement between forecast and observed levels. A magnetic activity index $D R X$ (being the daily mean of the 24 one-hour ranges in the north component) calculated for each of three zones-polar cap, auroral, and sub-auroral-demonstrates the distinctly different characters of these magnetic zones. Accordingly, values of $D R X$ are forecast for each of these three zones. Recurrent patterns of solar phenomena and their observed interactions with the geomagnetic field form the basis of the forecasting technique. Coronal holes predominate as sources of recurrent magnetic activity. During an interval in $1984-1985,88 \%$ of solar coronal holes were followed by an increase in geomagnetic activity.
\end{abstract}

\section{Introduction}

In recent years, forecasting of geomagnetic activity has been in increasing demand by a variety of user groups, including the exploration industry, communications agencies, power utilities, and scientific researchers. A number of institutes around the world, concerned with various aspects of solar-terrestrial relations, provide forecasts of solar activity and of related geophysical phenomena. The Earth Physics Branch (now amalgamated with the Geological Survey of Canada), of the Canadian Department of Energy Mines and Resources, has provided a geomagnetic activity forecast service since 1974 . Initially, only medium-term (27-day) forecasts were issued but since 1976, in addition, short-term forecasts up to 3 days have been provided. In these forecasts, three levels of magnetic activity were defined (quiet, unsettled, and active) and the daily prediction consisted of one of these levels. It was at that time already recognized that each of these three defined levels required a separate quantitative description for the subauroral, auroral, and polar cap regions (HRUSKA, 1979). Other formats have been used by other institutes: for example, the Space Environment Services Center (SESC) in Boulder, Colorado, has used a six-level

Geological Survey of Canada Contribution No. 41186. 
forecast (SESC, 1985). Recently, research into probability forecasting has also been reported (JOSELYN, 1984).

The level and character of magnetic activity vary greatly with geomagnetic latitude (e.g. Whitham et al., 1960; MOE and NeBERGALL, 1969; CAMPBELL, 1973). To incorporate this latitudinal dependence into the geomagnetic activity forecasts, HRUSKA (1983) recommended the use of a three-zone forecast for the three dominant magnetic zones in Canada.

This paper will describe the development of new multi-zone forecasts of geomagnetic activity for Canada and their verification for the period 1984 to 1985 .

\section{The Character of the Magnetic Field over Canada}

Although magnetic field data of various kinds have been collected in Canada for three centuries, it is only since about the time of the International Geophysical Year (1957-58) that the complexity of the externally-driven time variations and their spatial distribution over Canada, and in fact at high latitudes in general, began to be fully appreciated (e.g., FELDSTEIN, 1986). Since that time, a network of magnetic observatories has been expanded across the country (JANSEN VAN BEEK et al., 1986) and several chains of magnetic variometer stations have existed for special campaigns (e.g. for the International Magnetospheric Study (IMS)). From such networks in Canada and elsewhere, and from satellites, a knowledge of the complex character of the high latitude magnetic field has emerged (for example, WHITHAM and LOOMER, 1957; LOOMER and WHITHAM, 1963; LOOMER and JANSEN VAN BEEK, 1969; AKASOFU, 1977; AKASOFU and KAMIDE, 1980).

Because solar activity is recognized as the primary source of external magnetic activity, BARTELS (1934) introduced a useful method of displaying magnetic character indices, based on the solar rotation period of 27 days, thus permitting the ready recognition of recurrent phenomena. This is a natural physical time interval in the context of this study.

WHITHAM et al. (1960) discussed the relative merits of various measures of magnetic activity at high latitudes and concluded by selecting the hourly ranges in magnetic field components for their studies. Other more sophisticated indices have been developed, such as the $A E$ index, but in general they are not available on a timely basis and often require data from a number of stations. For the present purpose, a simple index was required that provided a measure of the daily activity in each zone and that was readily calculable in a timely fashion. Thus, a daily character index $D R X$ has been defined as the average of the 24 hourly ranges in the $X$ (north) component, to be determined from representative observatories for all the zones over Canada.

Three dominant zones having different magnetic field behaviour (sub-auroral, auroral, and polar cap) exist over Canada, with boundaries defined by physical features in the magnetosphere (AKASOFU, 1968). Although the actual positions of the instantaneous boundaries between the zones are continually changing (AKASOFU, 1968), in simple terms the zones can be described relative to geomagnetic latitudes: sub-auroral, $50^{\circ}$ to $60^{\circ} \mathrm{N}$; auroral, $60^{\circ}$ to $75^{\circ} \mathrm{N}$; polar cap, above $75^{\circ} \mathrm{N}$. For the 
present study, we found that the $D R X$ values from the stations in each zone were normally sufficiently close to one another that we were able to choose one representative observatory for each zone: Ottawa-sub-auroral; Fort Churchillauroral; Resolute Bay-polar cap (Fig. 1).

Figure 2 illustrates the diversity in magnetic activity in these zones. This figure is based on an analysis of the index $D R X$; the observed $D R X$ levels were sorted into bins, whose sizes are shown in the middle of the figure. The bin sizes were chosen by considering the typical high values of $D R X$ in a zone and subdividing into eight bins. For each Bartels rotation, the percentage occurrences of the bins are shown in a vertical bargraph, labelled with the Bartels rotation number. The equinoxes, summer, and winter are indicated. Results from two intervals of time are displayed in this fashion. First, twelve rotations in 1982 were chosen, near a peak in the 11 year cycle of magnetic activity, and second, twenty-two rotations during $1984-86$ in a quieter part

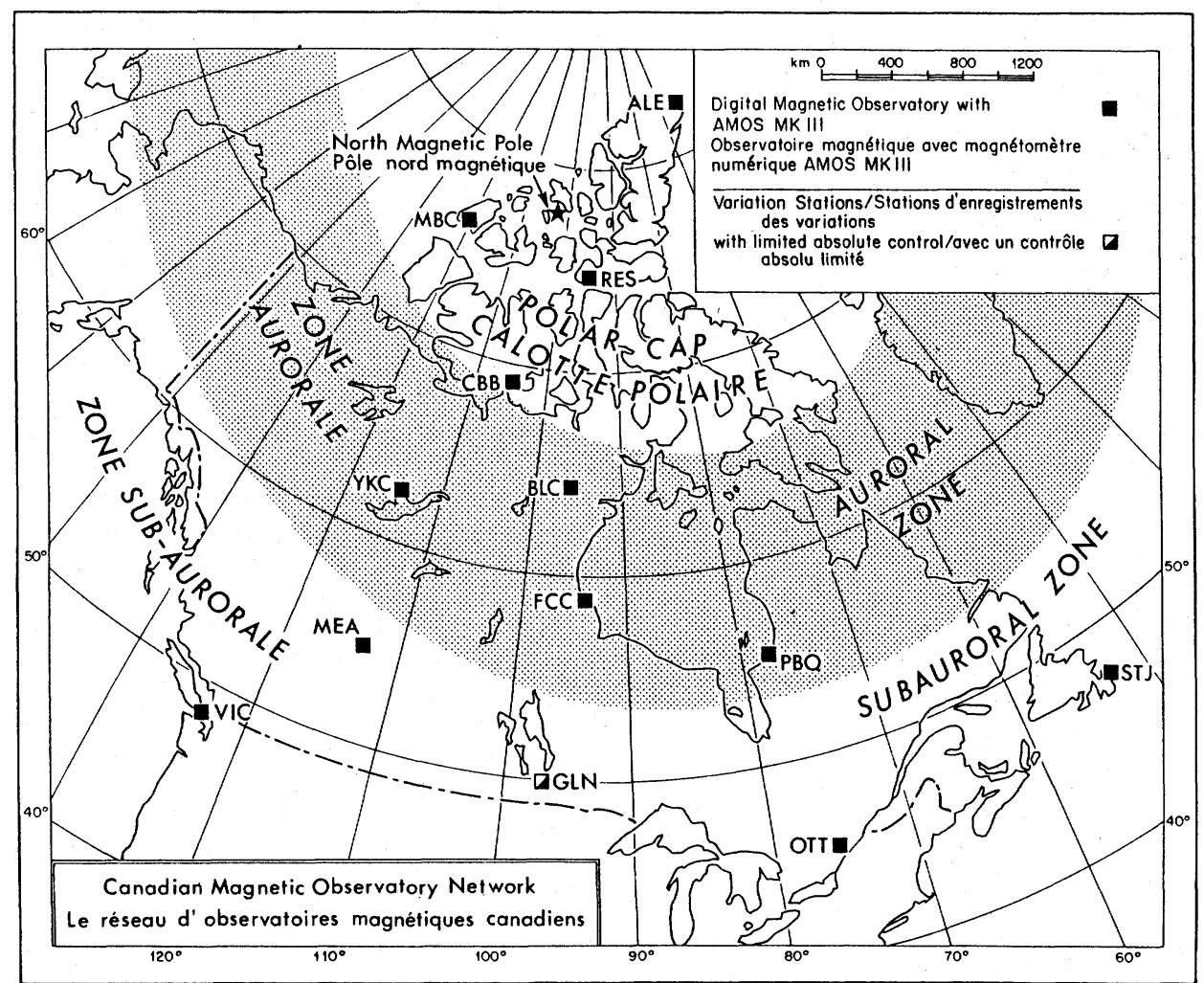

Fig. 1. Map showing approximate positions of the three major magnetic zones over Canada, as discussed in the text, and also the locations of the Canadian Magnetic Observatories. 

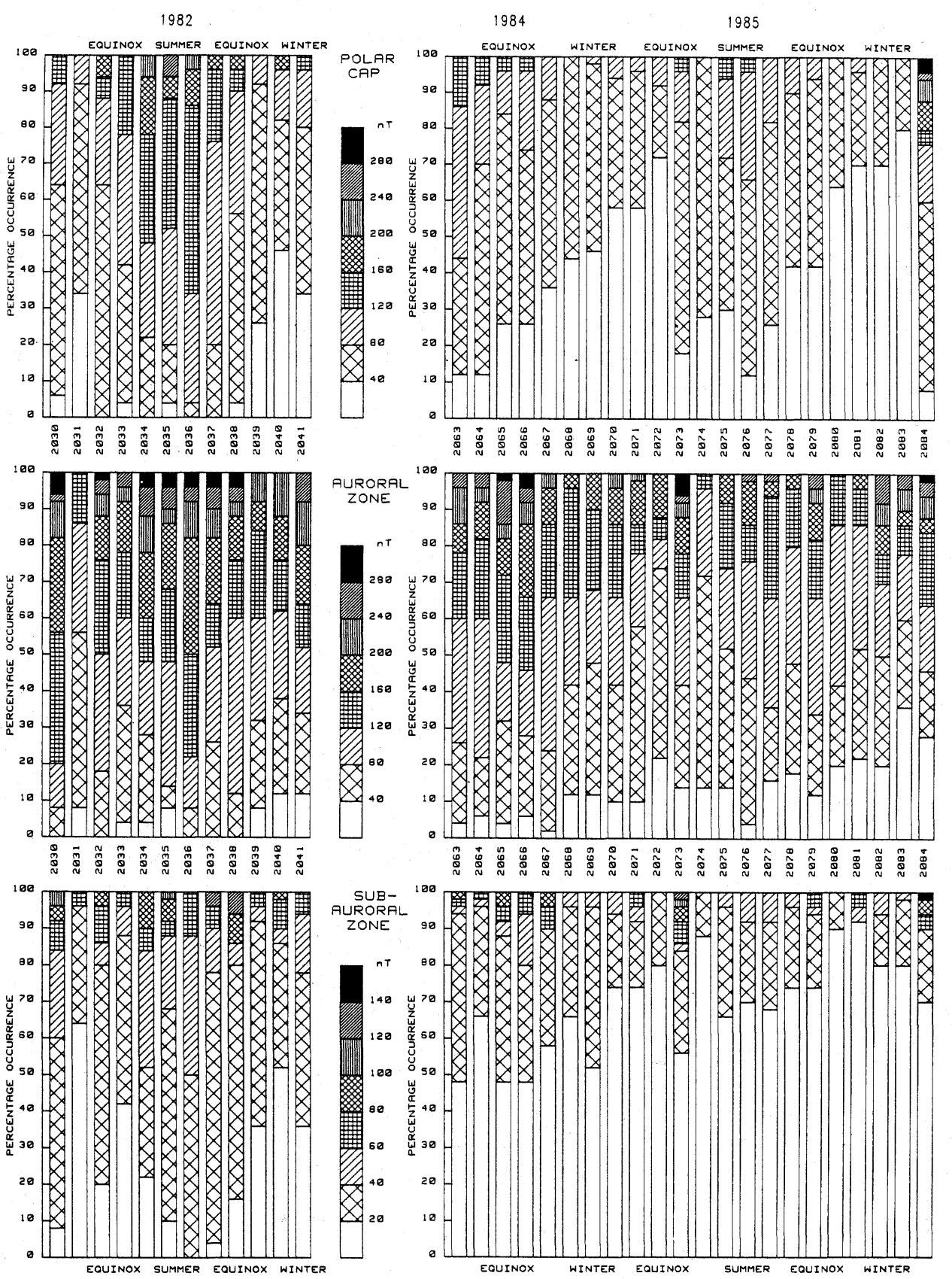

Fig. 2. Bar-graphs showing the percentage occurrence of the various binned levels of magnetic activity during 27-day Bartels rotation intervals (Bartels numbers are noted along the abscissae) for each of the three magnetic zones, during an active period (in 1982) and a quiet period (1984-1986) of the 11-year cycle. The data shown are derived from the representative observatories, Ottawa, Fort Churchill and Resolute Bay, from each zone. 
of the 11 year cycle. Note the distinct differences in character (a) between the 1982 interval and the 1984-86 interval, and (b) from zone to zone. In the polar cap during the later interval there is a striking 'sawtooth' pattern in activity, which becomes steadily quieter during the winter, but erupts into higher activity during the spring equinox. In the 1982 active interval, this sawtooth pattern is not seen, but rather there is a general increase in activity during the summer. In the auroral zone, higher levels of activity predominate. There is little systematic variation during 1982 but during 1984-86 there is an indication of high activity peaking in the equinoxes. Again, in the subauroral zone, there is little systematic variation at the higher activity levels during 1982 , though a decrease in very quiet conditions is seen during the summer. Once more, the higher activity levels are more common during equinoxes in 1984-86, although the field was predominantly quiet.

\section{The Basis of the Forecasting Process}

The external variations of the geomagnetic field are related in a complex way to solar activity. Two types of geomagnetic activity have been recognized (OL, 1971; SARGENT, 1985):

(1) Recurrent activity, including both very quiet and disturbed intervals, with a maximum of occurrence during the declining phase of the 11-year solar sunspot cycle;

(2) Sporadic activity, of disturbed intervals, with a maximum of occurrence during and around the peak of the solar sunspot cycle.

Coronal holes are currently considered to be recurrent solar sources of the high speed regions of the solar wind (KRIEGER et al., 1973) which often result in geomagnetic storms (SHEELEY et al., 1976). Coronal holes constitute one of the most significant structures in the solar corona (NOYES, 1982). Whereas the corona normally radiates large amounts of X-ray and ultraviolet emissions, coronal holes are large regions conspicuous for the near total absence of these emissions. However, coronal holes are almost undetectable in visible light. They are not permanent features of the solar corona, but wax and wane with lifetimes that may range from a few weeks to months. There are long-lived coronal holes in the polar regions, that sometimes grow in size with equatorward extensions. Other coronal holes form at lower heliolatitudes and over their lifespan often change their latitudinal position significantly. Coronal holes that are closer to the equator of the solar disc tend to produce high-speed solar wind streams that have a greater probability of reaching and coupling with the earth's magnetosphere. The delay between a coronal hole crossing the central meridian of the solar disc and the onset of geomagnetic activity depends on the speed of the solar wind.

Using a previous three-level characterization of magnetic activity (quiet: $K<3$; unsettled: $2<K<5$; or active: $K>4$ ), we have attempted to identify the solar source(s) associated with observed magnetic activity, using solar data from the SolarGeophysical Data Prompt Reports (NOAA). It was found that for the twenty-five magnetically active periods observed from March 1984 to September 1985, 52\% of

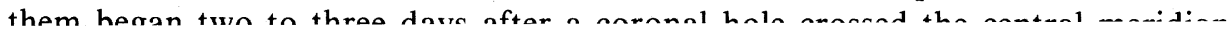


However, $20 \%$ of these may also have resulted from solar flares or disappearing filaments which occurred at about the same time.

Using the more definitive activity index $D R X$, described earlier, we have examined in more detail the effects of coronal holes on magnetic activity in the three magnetic zones over Canada. In doing this, the magnetic response was characterized by the maximum increase $\delta$ in the values of $D R X$ during the interval of five days following a solar event interpreted as a causative feature. This differs from the usual approach, in which the magnetic character itself must exceed a certain value to be counted in the analysis. Three groups were established: Group I, of 26 events, included coronal holes occurring alone; Group II, of 23 events, included coronal holes in combination with brilliant solar flares, or disappearing filaments; Group III, of 60 events, included solar flares or disappearing filaments alone or in combination. Table 1 contains the mean and median values of $\delta$ for increases in $D R X$ associated with each of the three Groups, for each zone. Significant increases in $\delta$ were considered to be those that exceeded half the bin-size for the zone (i.e. $10 \mathrm{nT}$ for sub-auroral, $20 \mathrm{nT}$ for the auroral and polar cap zones). Table 2 shows the percentage of occurrences of a particular Group that resulted in significant increases $\delta$ (by this criterion), for the three zones.

Table 1. Means and medians of $\delta$.

\begin{tabular}{lccc}
\hline & \multicolumn{3}{c}{ Magnetic zone } \\
\cline { 2 - 4 } Solar event & $\begin{array}{r}\text { Sub-auroral Auroral Polar cap } \\
\text { mean (median) }(\mathrm{nT})\end{array}$ \\
\hline $\begin{array}{l}\text { Group I } \\
\text { CH alone (26 events) }\end{array}$ & $24(17)$ & $105(96)$ & $46(42)$ \\
$\begin{array}{l}\text { Group II } \\
\text { CH + other (23 events) }\end{array}$ & $37(19)$ & $108(85)$ & $39(38)$ \\
$\begin{array}{l}\text { Group III } \\
\text { other (60 events) }\end{array}$ & $11(6)$ & $34(31)$ & $17(4)$ \\
\hline
\end{tabular}

Table 2. Percentage occurrence of significant $\delta$ associated with types of solar event.

\begin{tabular}{lccc}
\hline & $\begin{array}{c}\text { Sub-auroral } \\
(\%)\end{array}$ & $\begin{array}{c}\text { Auroral } \\
(\%)\end{array}$ & $\begin{array}{c}\text { Polar cap } \\
(\%)\end{array}$ \\
\hline $\begin{array}{l}\text { Group I } \\
\text { CH alone }\end{array}$ & 69 & 97 & 73 \\
$\begin{array}{l}\text { Group II } \\
\text { CH + other }\end{array}$ & 65 & 83 & 74 \\
$\begin{array}{l}\text { Group III } \\
\text { other }\end{array}$ & 27 & 60 & 30 \\
\hline
\end{tabular}


In this study of activity during the solar minimum (for the interval April 1984 to September 1985), it has been found that of the 49 reported coronal hole passages, $88 \%$ were followed within 2 to 5 days by a significant increase in magnetic activity in one or more magnetic zone.

Other features such as flares and disappearing filaments have been shown to be possible sources of geomagnetic disturbances (e.g. DODSON-PRINCE et al., 1978; JOSELYN and MCINTOSH, 1981; MiKHAILUTSA and GNEVYSHEV, 1985), but these are not normally recurring features and do not lend themselves directly to medium-and long-term forecasting.

The phenomenon 'opposite' to storms, namely, very quiet intervals, also shows a remarkable recurrent tendency (Hruska, in preparation). However, a causal link between these intervals and appropriate solar phenomena so far has not yet been established. Very quiet magnetic intervals can occur in spite of concurrent solar activity.

The preparation of a 27-day forecast is based on the recurrent activity. Available data on the solar features, including coronal holes, and on the past behaviour of the geomagnetic field are used. Past geomagnetic events are related where possible to observed solar phenomena. If the solar source is of a recurrent nature, e.g. a coronal hole, then the likelihood of its recurring again during the forecast period is assessed, taking into consideration the previous history of the source feature. In addition, terrestrial seasons are considered, since, as demonstrated in Fig. 2, seasonal variations in the level of the geomagnetic response to the solar activity are significant. The position in the 11-year solar cycle is a factor also-not always, however, working in the forecaster's favour. SARGENT (1985), for example, has shown how recurrent features predominate during the years around the minimum of solar activity, whereas they become obscured by sporadic activity during the years of solar maximum.

\section{Forecast Examples}

Examples of the three zone forecast are shown in Fig. 3; also included in the figure are occurrences of coronal holes, disappearing filaments and a solar flare. The forecast in Fig. 3(a) for rotation 2069 shows a good agreement between forecast and actual values of the index $D R X$ for all three zones. Two persistent coronal holes existed on the sun at this time giving rise to recurrent geomagnetic activity. The second example, rotation 2078, shows somewhat less agreement, particularly in the early part of September where magnetic activity failed to have the intensity that was forecast on the basis of previous events. Some events of a sporadic nature, and therefore not forecastable on the basis of recurrence, also occurred during this time interval.

\section{Forecast Verification}

A problem common to all forecasting is how to measure a forecast's accuracy. Many types of forecast now use a probabilistic form of expression. SANDERS (1963) 


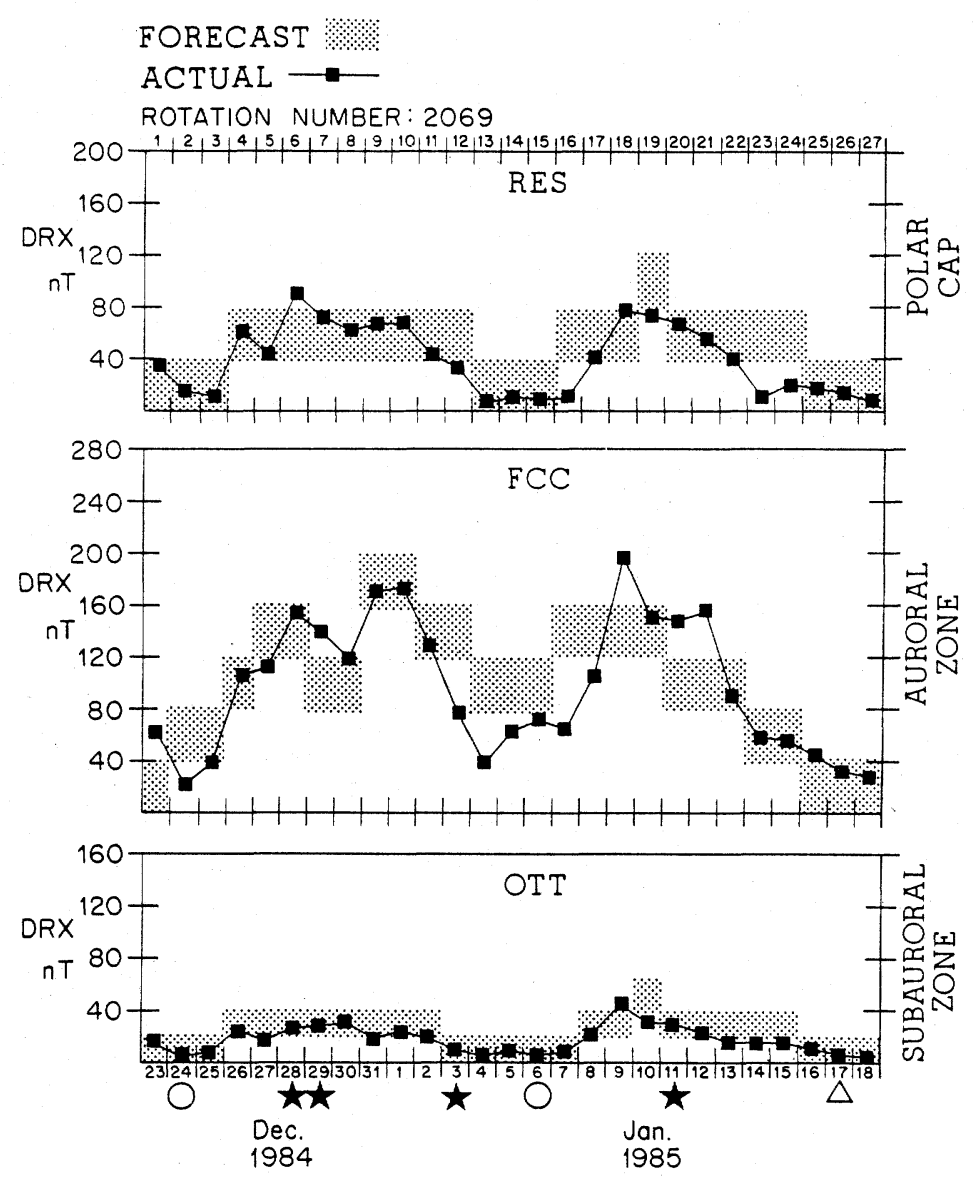

(a)

Fig. 3. Forecasts of the index $D R X$ for the three zones compared with observed values of $D R X$ from the representative observatories in these zones, (a) for Bartels rotation 2069, and (b) for Bartels rotation 2078. Coronal hole crossings of the solar central meridian are indicated by open circles; occurrences of disappearing filaments are shown by stars; a solar flare is indicated by an open triangle.

has discussed verification procedures applicable to probability forecasts.

The magnetic activity forecasts described here use a more direct categorical approach, forecasting an actual quantity. Though not always exactly analogous, some meteorological forecasts do bear similarities to magnetic activity forecasts and a number of attempts at verification techniques for the former have been explored in the literature (e.g. DOBR YSHMAN, 1972). For purposes of assessing the accuracy of the new magnetic forecasts, we have used the statistics known as the 'mean absolute deviation $M$ ' and the 'root mean square deviation $R$ ' defined thus: 


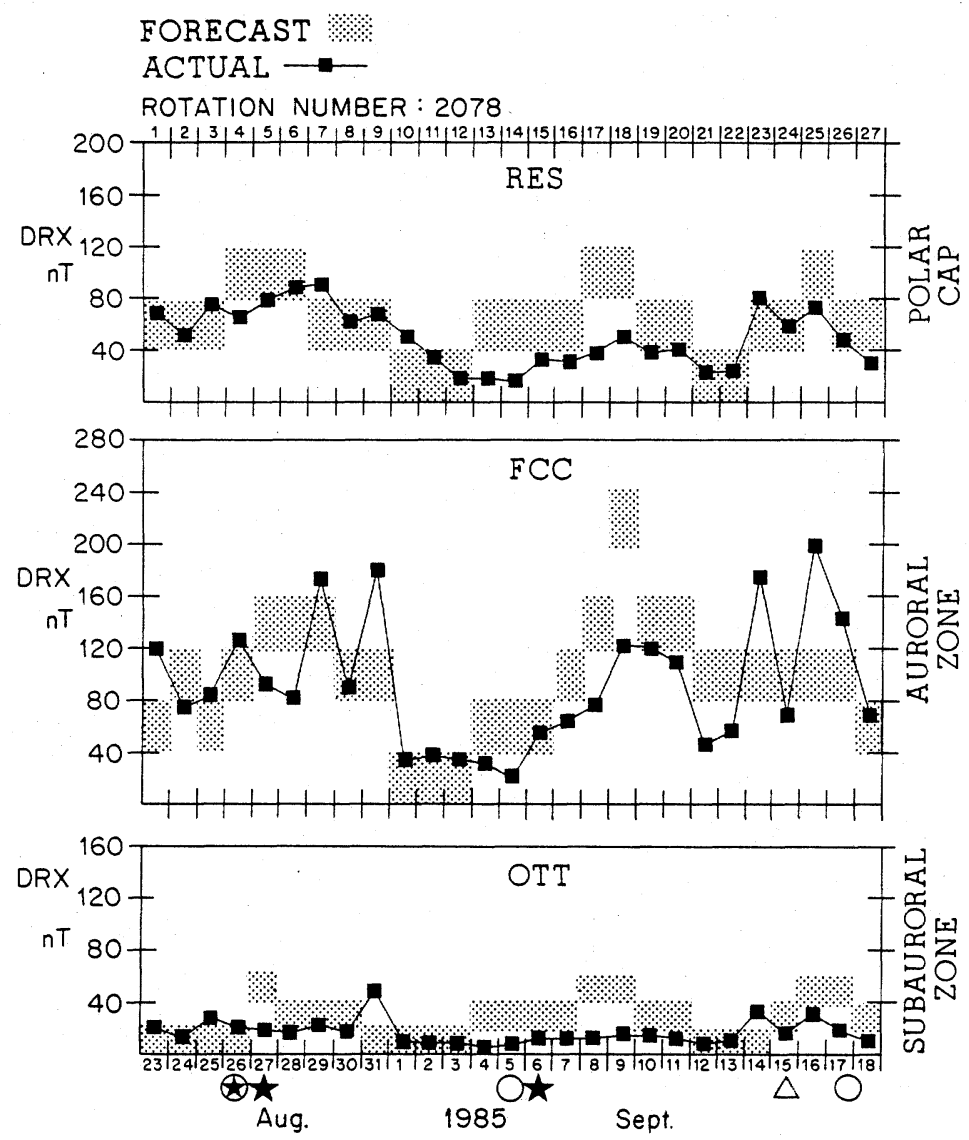

(b)

Fig. 3. (continued).

$$
M=\frac{\Sigma|f o r-o b s|}{N}, \quad R=\left[\frac{\sum(f o r-o b s)^{2}}{N}\right]^{1 / 2}
$$

where 'for' is the forecasted value, and 'obs' is the observed value.

These statistics have been applied to the complete sequence of medium-term forecasts (up to 27 days in advance) for about 460 days over 17 Bartels rotations in 1984-86, for each zone. In this case, the forecast and observed $D R X$ values were binned in the same manner as indicated in Fig. 2, and the statistics are shown in Table 3. One of the difficulties with these statistics is that there is no simple way of knowing what is a good value. Some form of normalization is required. To this end, we define: 
Table 3. Forecast verification statistics.

\begin{tabular}{lcccccccc}
\hline Zone & $\begin{array}{c}\text { Bin size } \\
(\mathrm{nT})\end{array}$ & \multicolumn{2}{c}{$M$} & \multicolumn{2}{c}{$R$} & $\begin{array}{c}\text { Mean } \\
\text { (bins) }\end{array}$ & $\begin{array}{c}{ }^{\circ} \mathrm{nbs} \\
(\mathrm{nT})\end{array}$ & $Q_{\mathrm{N}}$ \\
\hline Polar cap & 40 & 0.42 & 17 & 0.72 & 29 & 1.64 & 66 & 0.10 \\
Auroral & 40 & 0.93 & 37 & 1.40 & 56 & 2.75 & 110 & 0.20 \\
Sub-auroral & 20 & 0.45 & 9 & 0.79 & 16 & 1.33 & 27 & 0.11 \\
\hline
\end{tabular}

$$
Q_{\mathrm{N}}=\frac{R}{\left[1 / N \Sigma(\operatorname{maxbin}-1)^{2}\right]^{1 / 2}}=\frac{R}{(\operatorname{maxbin}-1)}
$$

where 'maxbin' is the number of bins, or subdivisions, available.

$Q_{\mathrm{N}}$ has a range from 0 to 1 , values closer to 0 representing better forecasts. Of course, the choice of the number of bins has a major influence on the value of $Q_{\mathrm{N}}$. In

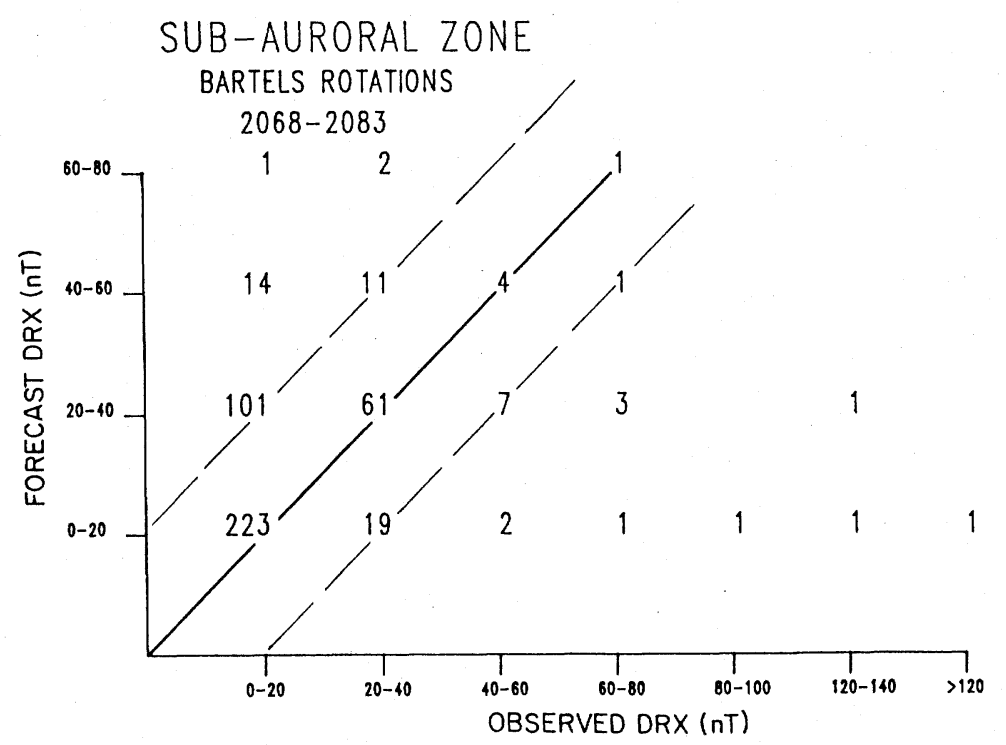

(a)

Fig. 4. Correlation diagrams between forecast and observed values of $D R X$ for (a) sub-auroral zone, (b) auroral zone, and (c) polar cap. The $D R X$ values were binned in the same manner as indicated in Fig. 2 ; the numbers in the diagrams indicate the numbers of occurrences at each grid point. The lines at $45^{\circ}$ through the origin indicate perfect correlations; the parallel lines include those forecasts that were in error by one bin. 


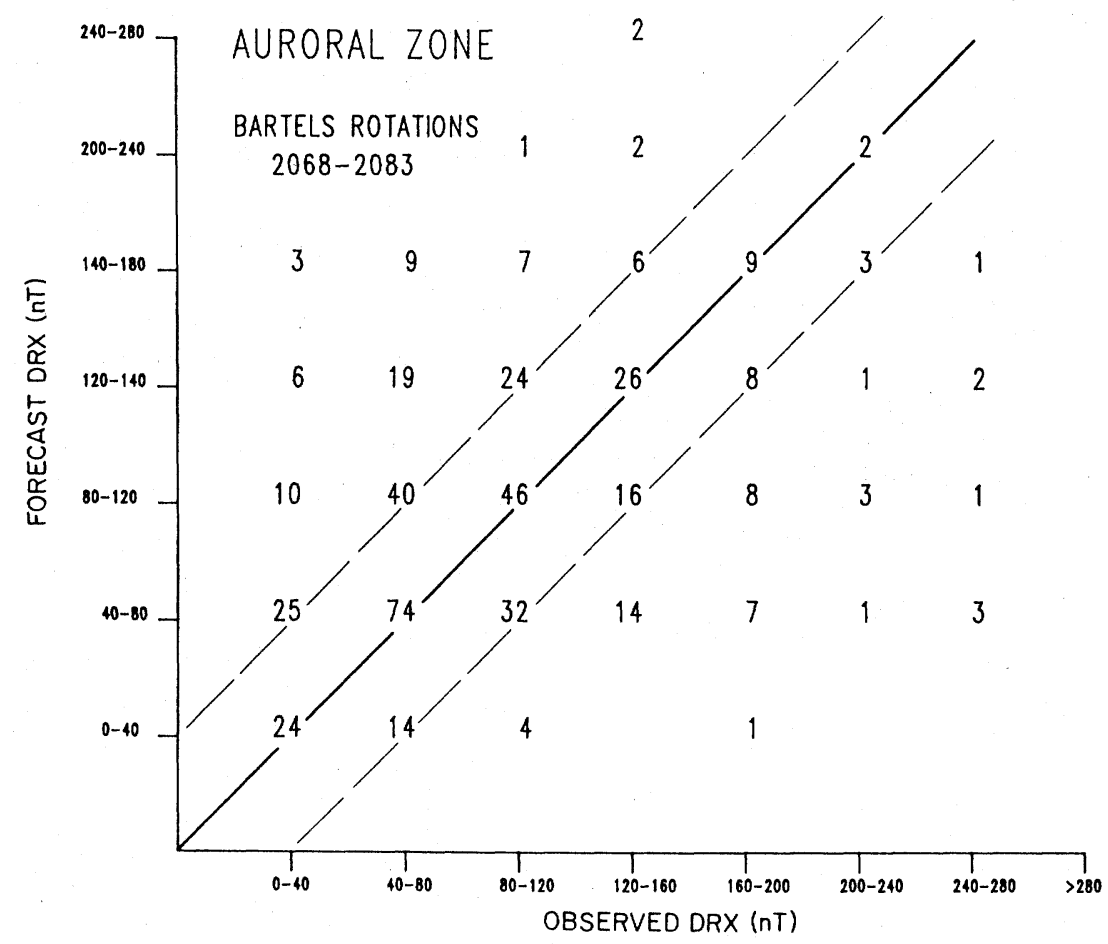

(b)

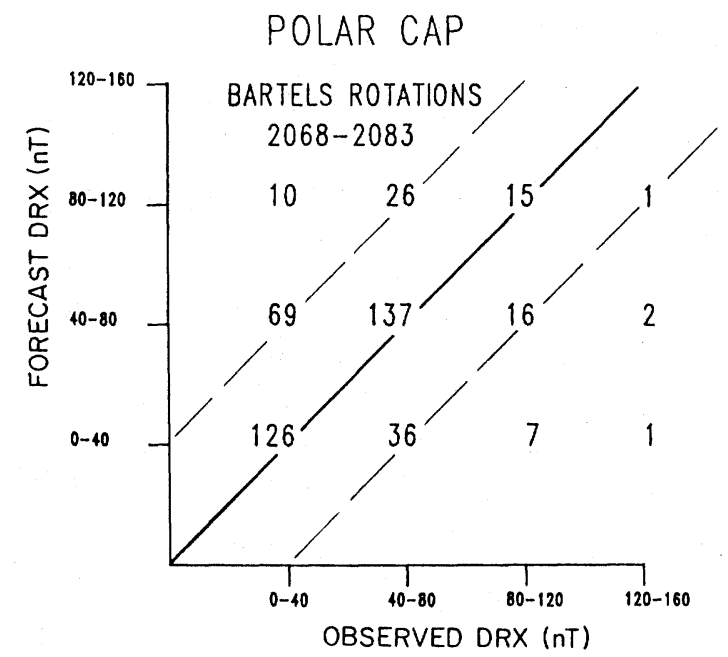

(c)

Fig. 4. (continued). 
this study, the number of bins (eight) was chosen on the basis of the data shown in Fig. 2 . So long as we continue to use this system then we have a consistent measure for our forecasts. It could, however, be readily adapted to another bin size and number. As an example to show the meaning of $Q_{\mathrm{N}}$, consider that all forecasts were in error by $1 \mathrm{bin}$. In that case, $R$ equals 1 , and $Q_{\mathrm{N}}$ equals 0.14 . Again, consider the case when all but one of 400 forecasts are correct, but the one is in error by 7 bins. Here, $R$ equals 0.35 , and $Q_{\mathrm{N}}$ equals 0.05 . The values of $Q_{\mathrm{N}}$ for the three zones during the study interval are also given in Table 3.

Figure 4 shows in a graphical form the extent of correlation between forecasted and observed binned mean ranges $D R X$. The numbers on the graphs indicate the numbers of occurrences at each grid point. The lines at $45^{\circ}$ through the origin indicate perfect correlations; the two parallel lines in each graph indicate those instances where the forecast was in error by only one bin. These displays readily show the incidence of correct or close forecasts, and also show instances when unforseen high disturbances occurred (when ' $o b s$ ' $\gg$ 'for'; these generally correspond to non-recurrent solar events). Another point to be noted is the rather large number of forecasts that are one bin higher than the observed bin, particularly at the lower levels. This indicates a forecaster bias during the learning process, often when the forecaster expected an event to recur with a particular intensity but which turned out to be weaker than in the previous rotation, or non-existent.

\section{Conclusions}

Experience has shown that the earlier use of a single forecast format for high latitudes was inadequate to characterize the diversity of magnetic field behaviour in the different zones. A new forecasting approach has been developed that aims to anticipate the level of magnetic activity in three major magnetic zones over Canada. However, as in earlier formats, the main technique used in producing these new forecasts relies on the recognition of recurrent features in the solar activity and their interactions with the magnetosphere. The new forecast scheme shows good agreement with actual activity, although sporadic, non-recurrent events do cause disagreements on occasion. The forecasts for the sub-auroral and polar cap zones were in the correct bin on $64 \%$ and $62 \%$ of the days, respectively, using the bin sizes as defined earlier. However, the activity in the auroral zone is more variable, and although only $40 \%$ of the forecasts were in the correct bin, $77 \%$ were within 1 bin of the observed bin. A parameter $Q_{\mathrm{N}}$, having a scale of 0 to 1 , has been defined, with lower values indicating better forecasts; for the study interval, $Q_{\mathrm{N}}=0.10$ for polar cap, $Q_{\mathrm{N}}=0.20$ for auroral zone, $Q_{N}=0.11$ for sub-auroral zone. This study indicates that the concept of multizone forecasting is correct, but that further adjustments to the technique are required. In fact, the application of linear prediction filter techniques to previous magnetic data (in the form of $D R X$ values) is being investigated for assisting in the production of forecasts (LAM, 1987).

During the period of development of this new forecast format, coronal holes have been the most reliable source of recurrent enhanced activity. Of the 49 passages 
of coronal holes reported, $88 \%$ were associated with significant increases in magnetic activity. The analysis has indicated that the responses to the passage of a coronal hole across the solar disc vary from one magnetic zone to another. Very quiet periods also show strong recurrent tendencies. These two types of feature form the basis for the forecasting process.

We would like to express our thanks to H.-L. Lam, L. R. Newitt, J. K. Walker and G. V. Haines for their constructive comments and suggestions during the preparation of this paper.

\section{REFERENCES}

AKasofu, S.-I., Polar and Magnetospheric Substorms, 280 pp., D. Reidel Publishing Company, Holland, 1968.

AKasofu, S.-I., Physics of Magnetospheric Substorms, 599 pp., D. Reidel Publishing Company, Holland, 1977.

AKASOFU, S.-I. and Y. KAMIDE, Recent progress in studies of magnetospheric storms and substorms, $J$. Geomag. Geoelectr., 32, 585-615, 1980.

Bartels, J., Twenty-seven day recurrences in terrestrial-magnetic and solar activity, 1923-33, Terr. Magn., 39, 201-202, 1934.

CAMPBELL, W. H., Spectral composition of geomagnetic field variations in the period range of $5 \mathrm{~min}$ to $2 \mathrm{hr}$ as observed at the earth's surface, Radio Science, 8, 929-932, 1973.

Dobryshman, E. M., Review of forecast verification techniques, World Meteorological Organization, Geneva, Technical Note No. 120, 1-52, 1972.

Dodson-Prince, H. W., E. R. Hedeman, and O. C. Mohler, Study of geomagnetic storms and solar flares in the years of increasing solar activity, cycles 19 and 20 (1955-1957, 1965-1968), Air Force Geophysics Laboratory, USAF Hanscomb AFB, Mass., Report AFGL-TR-78-0267, 1978.

FELDSTEIN, Y. I., A quarter of a century with the auroral oval, EOS Transactions of American Geophysical Union, Vol. 67, No. 40, p. 761, 1986.

HRUSKA, J., Forecasts of geomagnetic activity by Ottawa magnetic observatory: their reliability and application, in Proceedings of International Solar-Terrestrial Predictions Workshop, edited by R. F. Donnelly, Boulder, Vol. 1, pp. 398-405, 1979.

HRUSKA, J., Forecasts of geomagnetic activity, Division of Seismology and Geomagnetism, Earth Physics Branch, Ottawa, Internal Report 83-4, pp. 1-27, 1983.

JANSEN van Beek, G., R. L. Coles, and L. R. Newitt, Annual report for magnetic observatories-1984, Earth Physics Branch, EMR, Ottawa, Canada, Geomagnetic Series No. 30, 1986.

Joselyn, J. A., Proposed major format change to geomagnetic activity reports and forecasts produced by the SESC, Boulder Colorado, U.S.A., Geophys. Surv., 6, 419-424, 1984.

Joselyn, J. A. and P. S. MCINTOSH, Disappearing solar filaments: a useful predictor of geomagnetic activity, J. Geophys. Res., 86, 4555-4564, 1981.

Krieger, A. S., A. F. Timothy, and E. C. Roelof, A coronal hole and its identification as the source of a high velocity solar wind stream, Solar Physics, 29, 505-525, 1973.

LAM, H.-L., Forecasts of geomagnetic activity in Canada by linear prediction filtering, J. Geomag. Geoelectr., this issue, 535-542, 1987.

LOOMER, E. I. and K. WhithaM, On certain characteristics of irregular magnetic activity observed at Canadian magnetic observatories during 1960, Publ. Dom. Obs., Ottawa, 27, 75-81, 1963.

LOOMER, E. I. and G. JANSEN VAN BEEK, The effect of the solar cycle on magnetic activity at high latitudes, Publ. Dom. Obs., Ottawa, 37, 169-180, 1969.

Mikhailutsa, V. P. and M. N. Gnevyshev, The solar causes of geomagnetic disturbances, Solar Physics, 98, 387-393, 1985. 
Moe, K. and D. Nebergall, Variation of geomagnetic disturbance with latitude, J. Geophys. Res., 74, 1305-1307, 1969.

Noyes, R. W., The Sun-Our Star, 263 pp., Harvard University Press, Cambridge, Mass., 1982.

OL, A. I., Physics of the 11-year variation of magnetic disturbances, Geomag. Aeron., 11, 549-551, 1971.

SANDERs, F., On subjective probability forecasting, J. Appl. Meteorol., 2, 191-201, 1963.

SARGENT III, H. H., Recurrent geomagnetic activity: evidence for a long-lived stability in the solar wind, $J$. Geophys. Res., 90, 1425-1428, 1985.

SESC, Preliminary report and forecast of solar geophysical data, NOAA-USAF Space Environment Services Center, Boulder, Colorado, Descriptive Text, SESC PRF 511, 18 June, 1985.

SheEley, Jr., N. R., J. W. HARVEY, and W. C. FeldMan, Coronal holes, solar wind streams, and recurrent geomagnetic disturbances: 1973-1976, Solar Physics, 49, 271-278, 1976.

Whitham, K. and E. I. Loomer, Characteristics of magnetic disturbance at the Canadian Arctic observatories, Publ. Dom. Obs., 18, No. 12, 1957.

Whitham, K., E. I. Loomer, and E. R. Niblett, The latitudinal distribution of magnetic activity in Canada, J. Geophys. Res., 65, 3961-3974, 1960. 